\title{
Discrete Sliding Mode Control for Hypersonic Cruise Missile
}

\author{
Yong Hua Fan, Peng Peng Yan, Fan Wang, and Hong Yang Xu \\ College of Astronautics, Northwestern Polytechnical University, Xi'an 710072, China \\ Correspondence should be addressed to Peng Peng Yan; ypp328@163.com
}

Received 24 December 2015; Revised 18 February 2016; Accepted 7 March 2016

Academic Editor: Driss Boutat

Copyright (C) 2016 Yong Hua Fan et al. This is an open access article distributed under the Creative Commons Attribution License, which permits unrestricted use, distribution, and reproduction in any medium, provided the original work is properly cited.

\begin{abstract}
A discrete variable structure control (DVSC) with sliding vector is presented to track the velocity and acceleration command for a hypersonic cruise missile. In the design an integrator is augmented to ensure the tracking with zero steady-state errors. Furthermore the sliding surface of acceleration is designed using the error of acceleration and acceleration rate to avoid the singularity of control matrix. A proper power rate reaching law is utilized in this proposal; therefore the state trajectory from any initial point can be driven into the sliding surface. Besides, in order to validate the robustness of controller, the unmolded dynamic and parameter disturbance of the missile are considered. Through simulation the proposed controller demonstrates good performance in tracking velocity and acceleration command.
\end{abstract}

\section{Introduction}

Recently hypersonic vehicle has attracted attention because of its potential military and civil application [1-7]. With the breakthrough of the scramjet and heat safeguard, the hypersonic vehicle can be developed into cruise missile to realize global strike. The control system of hypersonic cruise missile exhibits high performance of tracking both velocity command and guidance command. Moreover the model of hypersonic missile has unmolded dynamic and parameter disturbance. Thus it is a challenge for hypersonic missile autopilot design [8].

The exploration of the various design methods for hypersonic aircraft has been an active field. Matthew [9] reported an adaptive linear quadratic (ALQ) altitude and velocity tracking control algorithm for the longitudinal model of a generic air-breathing hypersonic flight vehicle. A nonlinear controller for an air-breathing hypersonic vehicle was designed by Fiorentini et al. [10]. The sliding variable structure control also shows its advantage in robustness to the disturbance [11-14]. Using this method, Xu et al. [4] presented an adaptive sliding mode control for hypersonic aircraft with nonlinear and model uncertainty. A high-order sliding mode control was investigated by Yang and Tian [11] and then a sliding variable structure controller is designed for a reentry hypersonic missile. Yu and Yuri [12] used high-order sliding surface to design a hypersonic missile controller with the trajectory restriction. In case there is uncertainty, neural network based hypersonic flight control research could be found in literatures [14-17].

Although these literatures have satisfactory effect for a continuous plant, the computer on board always works discontinuously, and thus controller on the basis of continuous system cannot offer the same performance by a digital computer with a certain sampling interval. This can cause the system instability [13, 18-20].

This paper presents a design method of hypersonic cruise missile autopilot using discrete variable structure control. The main task of autopilot is to track commands of the acceleration and velocity simultaneously. So the multiple sliding surfaces are designed (for both subsystems of acceleration and velocity). To track with zero steady errors, an integrator is introduced into them. In this paper, chattering is reduced by a proper design of power rate reaching law. The unmolded dynamics and uncertainties are also included to examine the robustness of the discrete variable structure controller.

This paper is organized as follows. The dynamics and discrete model of hypersonic cruise missile are given in Section 2. And the design of discrete variable structure controller is presented in Section 3. Then we conduct simulations and give the results to demonstrate the reasonability of our design. Finally, we make a conclusion in Section 5. 


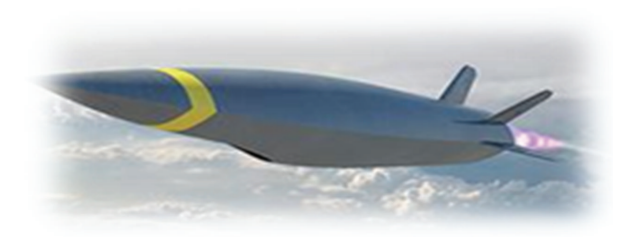

FIGURE 1: Configuration of hypersonic cruise missile.

\section{Model of Hypersonic Cruise Missile}

2.1. Description of Hypersonic Cruise Missile. The configuration of hypersonic cruise missile with scramjet is shown as in Figure 1.

The cowl of scramjet is arranged in the mandible of hypersonic cruise missile. The fore body is unsymmetrical, where the guidance system and warhead are located. And the lower fore body is the inlet of engine. Two elevatorailerons and two rudders are used to control pith, roll, and yaw movement. The thrust is adjusted by throttle. The longitudinal model of the cruise missile considered in this paper is

$$
\begin{aligned}
\dot{V} & =\frac{T \cos \alpha-D}{m}-g \sin \gamma, \\
\dot{\gamma} & =\frac{L+T \sin \alpha}{m V}-\frac{g \cos \gamma}{V}, \\
\alpha & =\theta-\gamma, \\
\dot{q} & =\frac{M_{y y}}{I_{y y}} \\
T & =\frac{1}{2} \rho V^{2} S C_{T}, \\
D & =\frac{1}{2} \rho V^{2} S C_{D}, \\
L & =\frac{1}{2} \rho V^{2} S C_{L}, \\
M_{y y} & =\frac{1}{2} \rho V^{2} S \bar{c}\left[C_{M}(\alpha)+C_{M}\left(\delta_{e}\right)+C_{M}(q)\right],
\end{aligned}
$$

where the expressions of aerodynamic coefficients are denoted by $C_{L}, C_{D}$, and $C_{M}$, respectively, and $C_{T}$ denotes thrust coefficient.

2.2. Discrete Model of Cruise Missile. Let $x=[\theta, \gamma, \alpha, V, q]^{T}$ be the state vector, and $u=\left[\delta_{t}, \delta_{e}\right]^{T}$ is the control vector. The longitudinal model of the cruise missile can be linearized:

$$
\begin{aligned}
& \dot{X}(t)=A X(t)+B U(t), \\
& X=[\theta, \gamma, \alpha, V, q]^{T},
\end{aligned}
$$

$$
\begin{aligned}
& U=\left[\delta_{t}, \delta_{e}\right]^{T}=\left[U_{1}, U_{2}\right]^{T}, \\
& A=\left[\begin{array}{ccccc}
0 & 0 & 0 & 0 & 1 \\
0 & V \cos \gamma & 0 & \sin \gamma & 0 \\
0 & \frac{g \sin \gamma}{V} & \frac{T+L^{\alpha}}{m V} & \frac{T^{V} \alpha+L^{V}}{m V} & 0 \\
0 & -\frac{g \sin \gamma}{V} & -\frac{T+L^{\alpha}}{m V} & -\frac{T^{V} \alpha+L^{V}}{m V} & 1 \\
0 & \frac{M_{y y}^{\gamma}}{I_{y y}} & \frac{M_{y y}^{\alpha}}{I_{y y}} & \frac{M_{y y}^{V}}{I_{y y}} & \frac{M_{y y}^{q}}{I_{y y}}
\end{array}\right] \text {, } \\
& B=\left[\begin{array}{cccccc}
0 & 0 & 0 & 0 & \frac{T^{\delta_{t}}}{m} & 0 \\
0 & 0 & \frac{L^{\delta_{e}}}{m V} & -\frac{L^{\delta_{e}}}{m V} & 0 & \frac{M_{y y}^{\delta_{e}}}{I_{y y}}
\end{array}\right]^{T},
\end{aligned}
$$

where $\theta, \gamma, \alpha, V$, and $q$ represent velocity, pitch angle, flight trajectory angle, angle of attack, and pitch rate, respectively. $\delta_{e}$ is the angle of elevator and $\delta_{t}$ is the control of throttle setting.

Assuming $T_{s}$ is the sampling interval of onboard computer and the plant is integrated in a sampling interval, the discrete state equations are

$$
\begin{aligned}
& X(k+1)=d A \cdot X(k)+d B \cdot U(k), \\
& X(k)=\left[\begin{array}{llllll}
\theta(k) & \gamma(k) & \alpha(k) & V(k) & q(k)
\end{array}\right]^{T}, \\
& d A=e^{A T_{s}}, \\
& d B=\left(\int_{0}^{T_{s}} e^{A t} d t\right) B, \\
& U(k)=\left[\begin{array}{ll}
\delta_{t}(k) & \delta_{e}(k)
\end{array}\right]^{T} .
\end{aligned}
$$

\section{Design of Variable Structure Controller}

The flight control system of hypersonic cruise missile is designed to track the commands of velocity and acceleration. Define the error of tracking as

$$
\begin{gathered}
a_{y}(k)-a_{y c}(k) \longrightarrow 0 \\
V(k)-V_{c}(k) \longrightarrow 0 .
\end{gathered}
$$

Let

$$
E=a_{y}+\dot{a}_{y} .
$$

$a_{y}$ is longitudinal acceleration, $\dot{a}_{y}$ is the rate of longitudinal acceleration, and the tracking error can be written as

$$
\begin{aligned}
& E(k)-E_{c}(k) \longrightarrow 0, \\
& V(k)-V_{c}(k) \longrightarrow 0 .
\end{aligned}
$$

The structure of the discrete control system of the hypersonic cruise missile is shown as in Figure 2. 


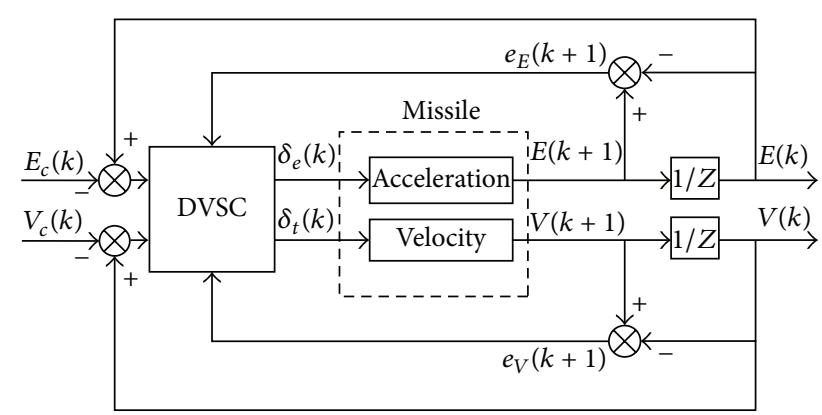

FIGURE 2: Structure of the discrete control system.

3.1. Design of Discrete Sliding Surface. Selecting multiple sliding surfaces,

$$
S(k+1)=\left[\begin{array}{l}
S_{1}(k+1) \\
S_{2}(k+1)
\end{array}\right]=\left[\begin{array}{l}
0 \\
0
\end{array}\right] .
$$

If the acceleration and velocity subsystem are chosen directly as the sliding surface,

$$
\begin{aligned}
S_{1}(k+1)= & {\left[a_{y}(k+1)-a_{y}(k)\right] } \\
& +c_{1}\left[a_{y}(k)-a_{y c}(k)\right],
\end{aligned}
$$

$$
S_{2}(k+1)=[V(k+1)-V(k)]+c_{2}\left[V(k)-V_{c}(k)\right],
$$

where $c_{1}$ and $c_{2}$ are the unknown parameters.

The acceleration can be written as

$$
a_{y}=V \dot{\gamma}
$$

Differentiating the acceleration expression, (17) yields

$$
\dot{a}_{y}=V \ddot{\gamma}=V(\ddot{\theta}-\ddot{\alpha})=V(\dot{q}-\ddot{\alpha}) .
$$

Substituting $\dot{q}$ and $\dot{\alpha}$ in terms of the sate equation (2) into (18) yields

$$
\dot{a}_{y}=-V A_{33} \dot{\alpha}
$$

Discretizing (19) yields

$$
\begin{gathered}
a_{y}(k+1)=-V A_{33} \alpha(k+1), \\
a_{y}(k)=-V A_{33} \alpha(k) .
\end{gathered}
$$

Substituting (20) and $V(k+1)$ in terms of the sate equation (7) into (15) and (16), respectively, yields

$$
S(k+1)=S_{X} X(k)+S_{Y} U(k)+S_{Z} \cdot
$$

To be simple, $d A(2,1)$ is written as $d A_{21}$; the other similar denotation is the same. Therefore the matrixes in (21) are

$$
\begin{aligned}
& S_{X}=\left[\begin{array}{ccccc}
-V A_{33} d A_{31} & -V A_{33} d A_{32} & -V A_{33}\left(d A_{33}+c_{1}-1\right) & -V A_{33} d A_{34} & -V A_{33} d A_{35} \\
d A_{41} & d A_{42} & d A_{43} & d A_{44}+c_{2}-1 & d A_{45}
\end{array}\right], \\
& S_{Y}=\left[\begin{array}{cc}
-V A_{33} d B_{31} & -V A_{33} d B_{32} \\
d B_{41} & d B_{42}
\end{array}\right] \text {, } \\
& S_{Z}=\left[\begin{array}{l}
-c_{1} a_{y c}(k) \\
-c_{2} V_{c}(k)
\end{array}\right]
\end{aligned}
$$

For $d B_{31}=d B_{32}=0$, the matrix $S_{Y}^{-1}$ will not exist. Then, $U(k)$ cannot be achieved. Therefore the sliding surface cannot be chosen as (15). As long as the differential of state variable includes $\Delta \dot{q}$, the matrix $S_{Y}$ is invertible. From the derivative of (19), the second-order derivative of acceleration is

$$
\ddot{a}_{y}=-V A_{33}\left(A_{33} \dot{\alpha}+\dot{q}\right) \text {. }
$$

In the right-hand side of (23), $\Delta \dot{q}$ is included and the matrix $S_{Y}$ is invertible; the control law $U(k)$ can be obtained.

Thereby, the form of control variable is as follows:

$$
E=a_{y}+\dot{a}_{y}
$$

Differentiating (24), we have

$$
\dot{E}=\dot{a}_{y}+\ddot{a}_{y}=-V A_{33}\left[\left(A_{33}+1\right) \dot{\alpha}+\dot{q}\right] .
$$

In terms of discretization (25) is

$$
\begin{aligned}
& E(k)=-V A_{33}\left[\left(A_{33}+1\right) \alpha(k)+q(k)\right], \\
& E(k+1)-E(k)=-V A_{33}\left[\left(A_{33}+1\right)\right. \\
& \quad \cdot(\alpha(k+1)-\alpha(k))+(q(k+1)-q(k))] .
\end{aligned}
$$

Substituting the discrete state equations $\alpha(k+1)$ and $q(k+1)$ into (27) yields

$$
E(k+1)-E(k)=S_{E 1} X(k)+S_{E 2} U(k) .
$$

The elements of matrix $S_{E 2}$ are not all zeros.

Choosing the sliding surface of the acceleration subsystem,

$$
S_{1}(k+1)=b[E(k+1)-E(k)]+c\left[E(k)-E_{c}(k)\right],
$$

where $b$ and $c$ are designed parameters. 
To enable the system for tracking the command with zero steady-state errors, a discrete integrator is added in the sliding surface:

$$
\begin{aligned}
& S_{1}(k+1) \\
&= a S_{1}(k) \\
&+T_{s}\left\{b[E(k+1)-E(k)]+c\left[E(k)-E_{c}(k)\right]\right\},
\end{aligned}
$$

where $a$ is an unknown coefficient to enhance the performance of integrator. Let $a=a_{1}, T_{s} b=1$, and $T_{s} c=c_{1}$; rearranging the equation yields

$$
\begin{aligned}
S_{1}(k+1)= & a_{1} S_{1}(k)+[E(k+1)-E(k)] \\
& +c_{1}\left[E(k)-E_{c}(k)\right] \\
S_{1}(k+1)-S_{1}(k)=[ & {[E(k+1)-E(k)] } \\
& +c_{1}\left[E(k)-E_{c}(k)\right] \\
& -\left(1-a_{1}\right) S_{1}(k) .
\end{aligned}
$$

With solution of (31) and (32) for $E(k+1)-E(k)$ and $E(k)$ can be obtained. Substituting $E(k+1)-E(k)$ and $E(k)$ into (32), we have

$$
S_{1}(k+1)-S_{1}(k)=S_{X 1} X(k)+S_{Y 1} U(k)+S_{Z 1},
$$

where, $S_{X 1} \in R^{1 \times 6}, S_{Y 1} \in R^{1 \times 2}$, with $S_{X 1}, S_{Y 1}$, and $S_{Z 1}$ given by

$$
\begin{aligned}
& S_{X 1} \\
& =-A_{33} V\left[\begin{array}{c}
\left(A_{33}+1\right) d A_{31}+d A_{51} \\
\left(A_{33}+1\right) d A_{32}+d A_{52} \\
\left(A_{33}+1\right) d A_{33}+d A_{53}+\left(c_{1}-1\right)\left(A_{44}+1\right) \\
\left(A_{33}+1\right) d A_{34}+d A_{54} \\
\left(A_{33}+1\right) d A_{35}+d A_{55}+c_{1}-1
\end{array}\right], \\
& S_{Y 1}=-A_{33} V\left[\begin{array}{c}
\left(A_{33}+1\right) d B_{31}+d B_{51} \\
\left(A_{33}+1\right) d B_{32}+d B_{52}
\end{array}\right]^{T}, \\
& S_{Z 1}=-c_{1} E_{c}(k)-\left(1-a_{1}\right) S_{1}(k) .
\end{aligned}
$$

For the velocity subsystem, the sliding surface can be chosen as

$$
\begin{aligned}
S_{2}(k+1)= & a_{2} S_{2}(k)+[V(k+1)-V(k)] \\
& +c_{2}\left[V(k)-V_{c}(k)\right] \\
S_{2}(k+1)-S_{2}(k)= & {[V(k+1)-V(k)] } \\
& +c_{2}\left[V(k)-V_{c}(k)\right] \\
& -\left(1-a_{2}\right) S_{2}(k) .
\end{aligned}
$$

Substituting the discrete state equation $V(k+1)$ into (36) yields

$$
S_{2}(k+1)-S_{2}(k)=S_{X 2} X(k)+S_{Y 2} U(k)+S_{Z 2},
$$

where

$$
\begin{aligned}
& S_{X 2}=\left[\begin{array}{lllll}
d A_{41} & d A_{42} & d A_{43} & d A_{44}+c_{2}-1 & d A_{45}
\end{array}\right], \\
& S_{Y 2}=\left[\begin{array}{ll}
d B_{41} & d B_{42}
\end{array}\right], \\
& S_{Z 2}=-c_{2} V_{c}(k)-\left(1-a_{2}\right) S_{2}(k) \text {. }
\end{aligned}
$$

Solving (33) and (37), we have

$$
\left[\begin{array}{l}
S_{1}(k+1)-S_{1}(k) \\
S_{2}(k+1)-S_{2}(k)
\end{array}\right]=S_{X} X(k)+S_{Y} U(k)+S_{Z}
$$

where $S_{X}=\left[\begin{array}{l}S_{X 1} \\ S_{X 2}\end{array}\right], S_{Y}=\left[\begin{array}{l}S_{Y_{1}} \\ S_{Y 2}\end{array}\right], S_{Z}=\left[\begin{array}{l}S_{Z 1} \\ S_{Z 2}\end{array}\right]$.

3.2. Design of Discrete Variable Structure Controller. Choose the power rate reaching law as

$$
\dot{S}=-\varepsilon \operatorname{sgn} S-\delta S .
$$

The discrete form of (40) is

$$
S(k+1)-S(k)=-T_{s} \delta S(k)-\varepsilon T_{s} \operatorname{sgn} S(k),
$$

where $T_{s}, \varepsilon>0$, and $\delta>0$ denote sampling interval, reaching rate, and exponent of reaching rate, respectively. The monotone reaching condition of the discrete power rate reaching law is

$$
T_{s} \delta<1 .
$$

Therefore the reaching conditions of acceleration subsystem and velocity subsystem are

$$
\begin{aligned}
& S_{1}(k+1)-S_{1}(k)=-T_{s} \delta_{1} S_{1}(k)-\varepsilon_{1} T_{s} \operatorname{sgn} S_{1}(k), \\
& S_{2}(k+1)-S_{2}(k)=-T_{s} \delta_{2} S_{2}(k)-\varepsilon_{2} T_{s} \operatorname{sgn} S_{2}(k) . \\
& \text { Rearranging (43) yields }
\end{aligned}
$$

$$
\left[\begin{array}{l}
S_{1}(k+1)-S_{1}(k) \\
S_{2}(k+1)-S_{2}(k)
\end{array}\right]=-S_{n d 1} S(k)-S_{n d 2} \operatorname{sgn} S(k),
$$

where

$$
\begin{aligned}
& S_{n d 1}=\left[\begin{array}{cc}
T_{s} \delta_{1} & 0 \\
0 & T_{s} \delta_{2}
\end{array}\right], \\
& S_{n d 2}=\left[\begin{array}{cc}
\varepsilon_{1} T_{s} & 0 \\
0 & \varepsilon_{2} T_{s}
\end{array}\right] .
\end{aligned}
$$




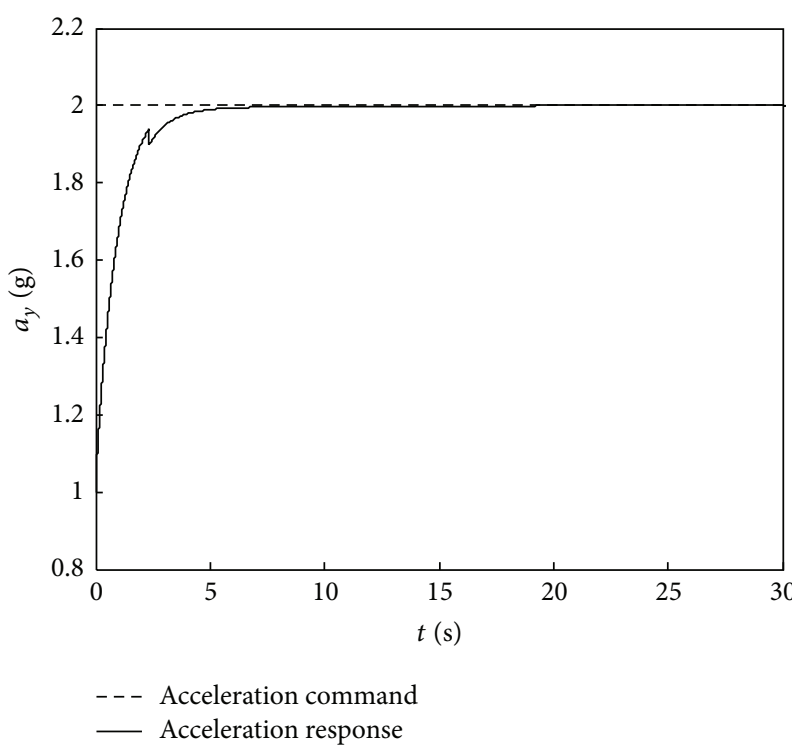

FIgURE 3: Response of acceleration.

With the solutions of (39) and (44), the control law in terms of vector is

$$
\begin{aligned}
& U(k)=\left(-S_{Y}\right)^{-1} \\
& \cdot\left[S_{X} X(k)+S_{Z}+S_{n d 1} S(k)+S_{n d 2} \operatorname{sgn} S(k)\right],
\end{aligned}
$$

where $\delta_{t}(k)=U_{1}(k), \delta_{e}(k)=U_{2}(k)$.

\section{Simulations}

Assuming the hypersonic cruise missile flight height is $H_{0}=$ $25 \mathrm{Km}, V_{0}=6 \mathrm{Ma}$, the equilibrium condition can be given as

$$
\begin{aligned}
L_{0}+T_{0} \sin \alpha_{0} & =m g, \\
T_{0} \cos \alpha_{0} & =D_{0}, \\
M_{z 0} & =0 .
\end{aligned}
$$

From solution of (47), the trim angle of attack $\alpha_{0}=$ $3.3420^{\circ}$, the trim angle of actuator $\delta_{e 0}=-0.6257^{\circ}$, and the trim of throttle setting $\delta_{t 0}=0.2418$. At this equilibrium point, the coefficient matrixes of the longitudinal linearization model are

A

$$
\begin{aligned}
& =\left[\begin{array}{ccccc}
0 & 0 & 0 & 0 & 1 \\
0 & 3.3 \times 10^{-11} & 0.0364 & 9.3 \times 10^{-7} & 0 \\
0 & -3.3 \times 10^{-11} & -0.0364 & -9.3 \times 10^{-7} & 1 \\
0 & -9.7489 & -21.3171 & 0 & 0 \\
0 & 0 & 0.3126 & 1.5 \times 10^{-18} & -0.0584
\end{array}\right], \\
& B=\left[\begin{array}{ccccc}
0 & 8.7 \times 10^{-5} & -8.7 \times 10^{-5} & 6.8603 & 0 \\
0 & 0 & 0 & 0 & 2.7378
\end{array}\right]^{T} .
\end{aligned}
$$

Suppose that the sample interval $T_{s}=0.005 \mathrm{~s}$; the state matrix in terms of discretization can be given:

$$
\begin{aligned}
& d A=\left[\begin{array}{ccccc}
1 & 7.3 \times 10^{-17} & 3.9 \times 10^{-6} & -6 \times 10^{-15} & 0.005 \\
0 & 1 & 1.8 \times 10^{-4} & 4.6 \times 10^{-9} & 4.6 \times 10^{-7} \\
0 & 1.1 \times 10^{-10} & 0.9998 & -4.6 \times 10^{-9} & 0.005 \\
0 & -0.0487 & -0.1066 & 1 & -2.7 \times 10^{-4} \\
0 & 5.8613 & 0.0016 & -3.6 \times 10^{-12} & 0.9997
\end{array}\right], \\
& d B=\left[\begin{array}{ccccc}
-5.7 \times 10^{-13} & 4.4 \times 10^{-7} & -4.4 \times 10^{-7} & 0.0343 & -3.4 \times 10^{-10} \\
3.4 \times 10^{-5} & 2.1 \times 10^{-9} & 3.4 \times 10^{-5} & -1.2 \times 10^{-6} & 0.0137
\end{array}\right]^{T} .
\end{aligned}
$$

Let $a_{1}=0.99, a_{2}=0.98, c_{1}=0.68, c_{2}=0.3, \delta_{1}=0.815$, $\delta_{2}=0.4, \varepsilon_{1}=0.005$, and $\varepsilon_{2}=0.005$; the control laws $\delta_{t}(k)$ and $\delta_{e}(k)$ can be obtained as in (40). The control system tracks a $2 \mathrm{~g}$ acceleration command and velocity command during cruising flight; the simulations are shown as in Figures 3-9.

The simulation results of discrete VSS controller law are shown as in Figures 3-9. In Figures 3 and 4, the solid lines are the command and the dashed lines are the response of the control system. It is observed that the hypersonic missile can track both acceleration and velocity command. The controller also has no steady error. The sliding surfaces of acceleration and velocity subsystem are also reaching zero with the power reaching rate as in Figures 5 and 6. The steady response of angle of attack is $6.5 \mathrm{deg}$. with $2 \mathrm{~g}$ maneuver acceleration, and the elevator and throttle setting response are shown as in Figures 7 and 8. With the discrete VSS controller, perfect tracking is achieved.

To validate the controller robustness to the model errors, the uncertainties of the model are considered as $|\Delta m| \leq 0.03$, $|\Delta I| \leq 0.05,|\Delta S| \leq 0.03,\left|\Delta C_{D}\right| \leq 0.15,\left|\Delta C_{L}\right| \leq 0.10$, $\left|\Delta C_{m}\right| \leq 0.10,|\Delta \rho| \leq 0.06$, and $\left|\Delta C_{T}\right| \leq 0.03$. The Monte Carlo simulation results of 100 times are shown as in Figures $10-16$. 


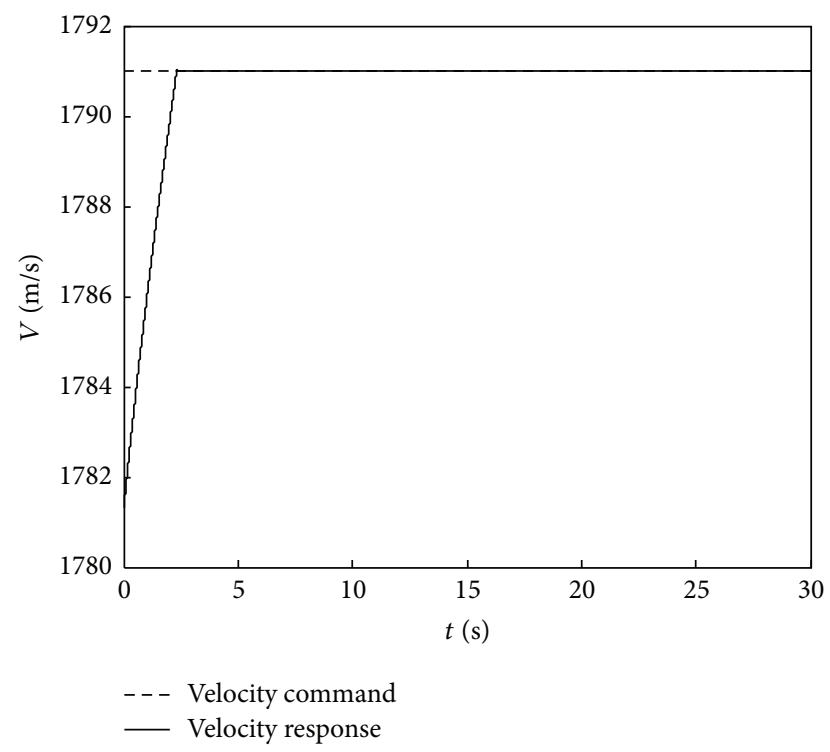

FIGURE 4: Response of velocity.

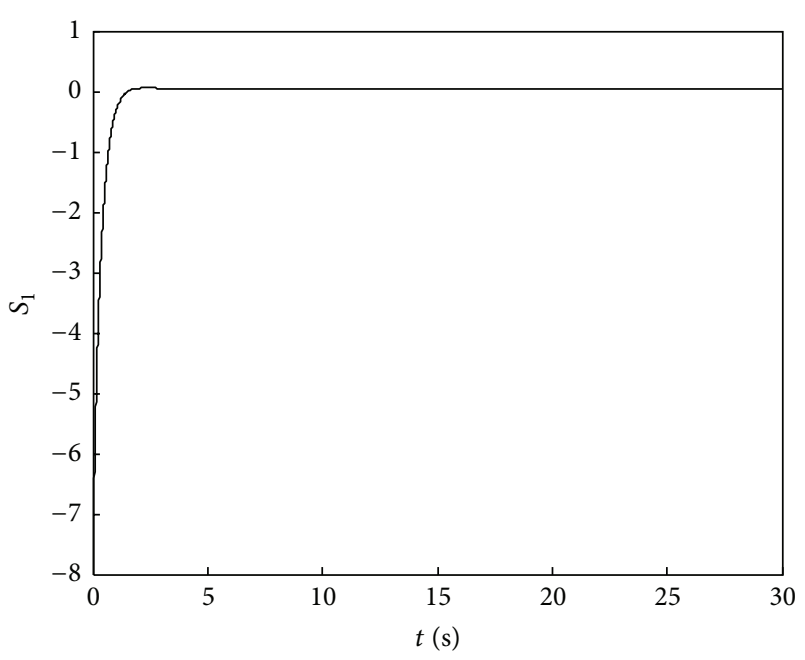

— Sliding surface of acceleration

FIGURE 5: Response of acceleration sliding surface.

It is shown that the controller has satisfactory performance to track the acceleration and velocity command with the model parameters uncertainties. For the errors of model mostly affect the acceleration of the missile, the sliding surface of acceleration has obvious response as shown in Figure 12. However, the velocity subsystem is less affected by the parameters disturbance as shown in Figure 13. The elevator is $-8 \sim 5 \mathrm{deg}$. with the uncertainties which is more great than result of the norm plant as shown in Figure 14.

\section{Conclusion}

In this paper a hypersonic cruise missile flight control system is designed using discrete variable structure controller to

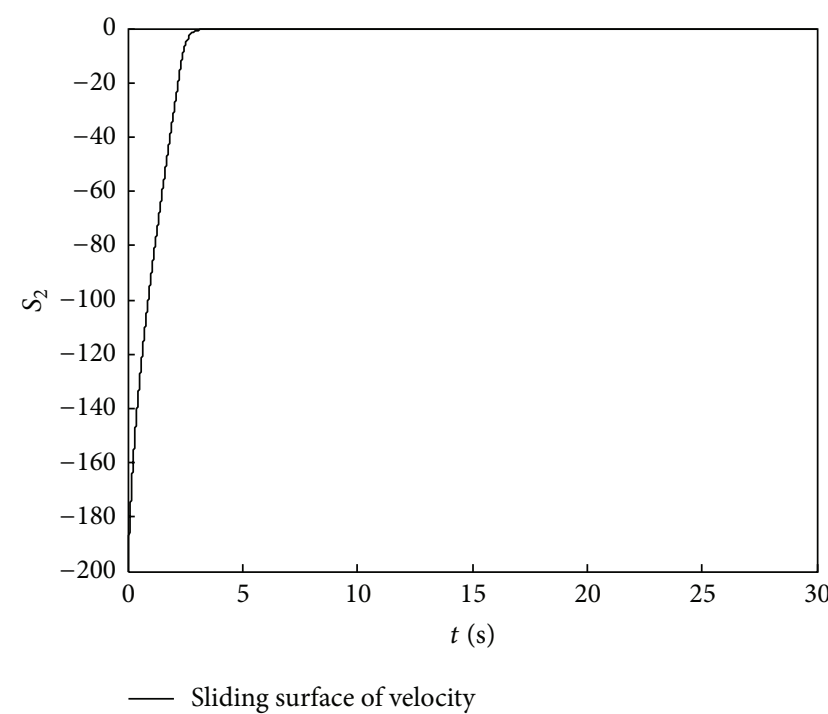

FIGURE 6: Response of velocity sliding surface.

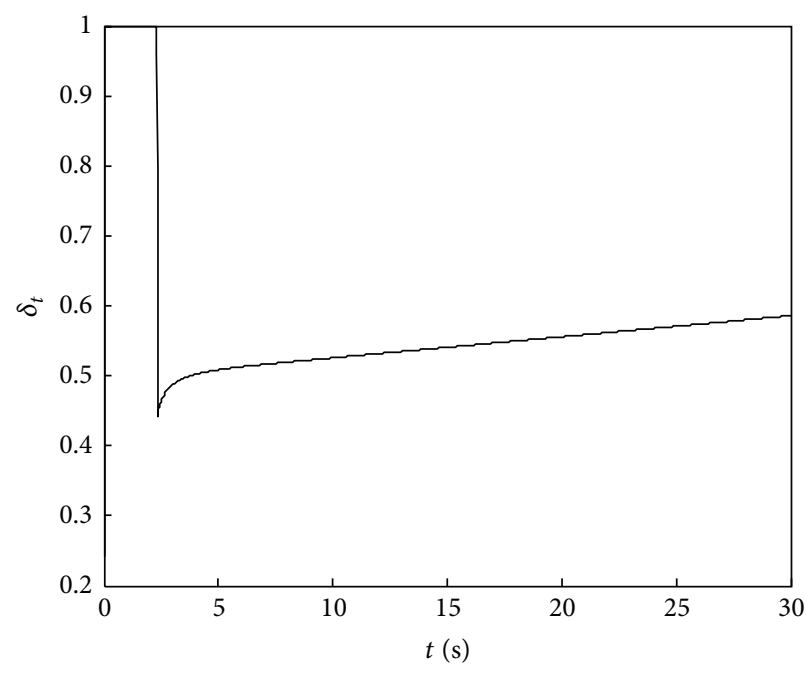

— Throttle

Figure 7: Response of throttle setting.

track the acceleration and velocity commands. An integrator is augmented to ensure that the tracking has no steady error. The multiple sliding surfaces are designed. And the control laws of acceleration and velocity subsystem are obtained. The uncertain missile plant with unmolded dynamic and parameter variations are considered. Simulation results demonstrate that the control system has good performance in tracking acceleration and velocity command and still has strong robustness to the uncertainties of model.

\section{Competing Interests}

The authors declare that they have no competing interests. 


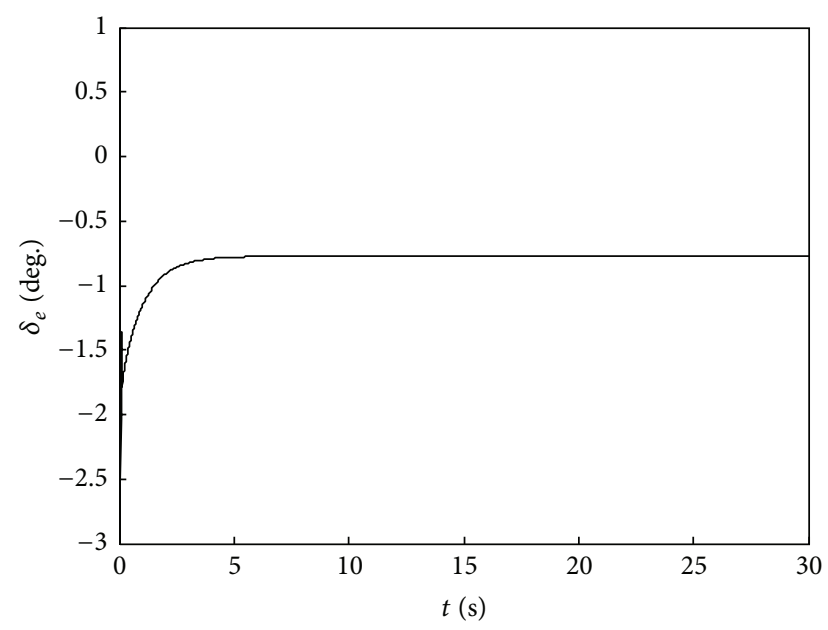

— Elevator

Figure 8: Response of elevator.

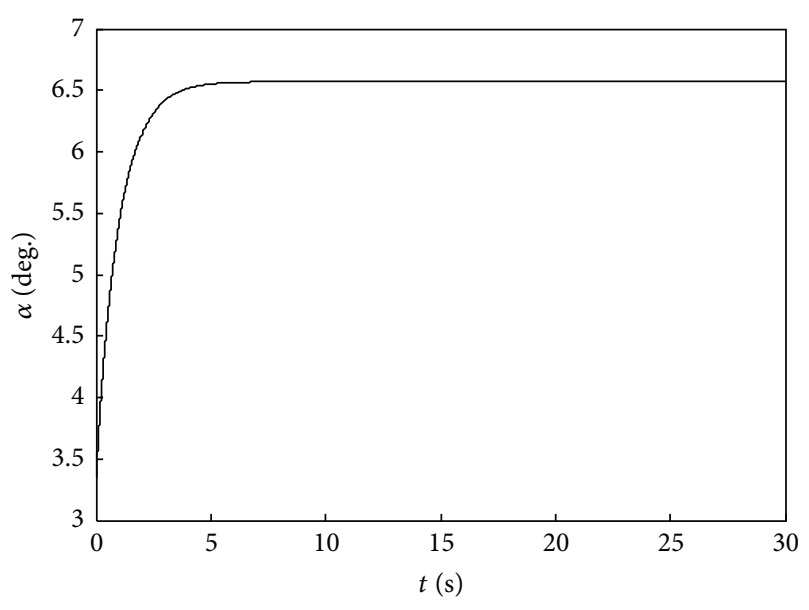

_ Angle of attack

FIGURE 9: Response of angle of attack.

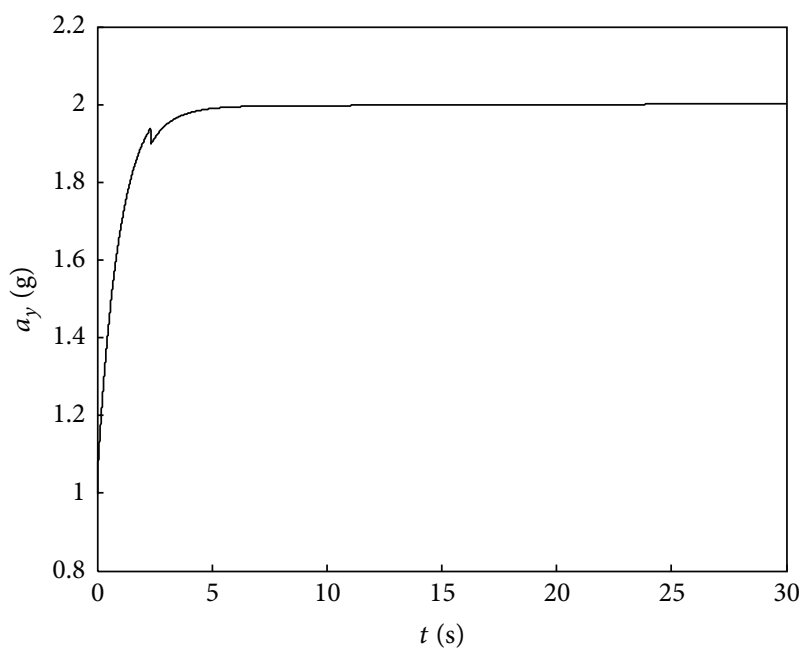

FIGURE 10: Results of acceleration.

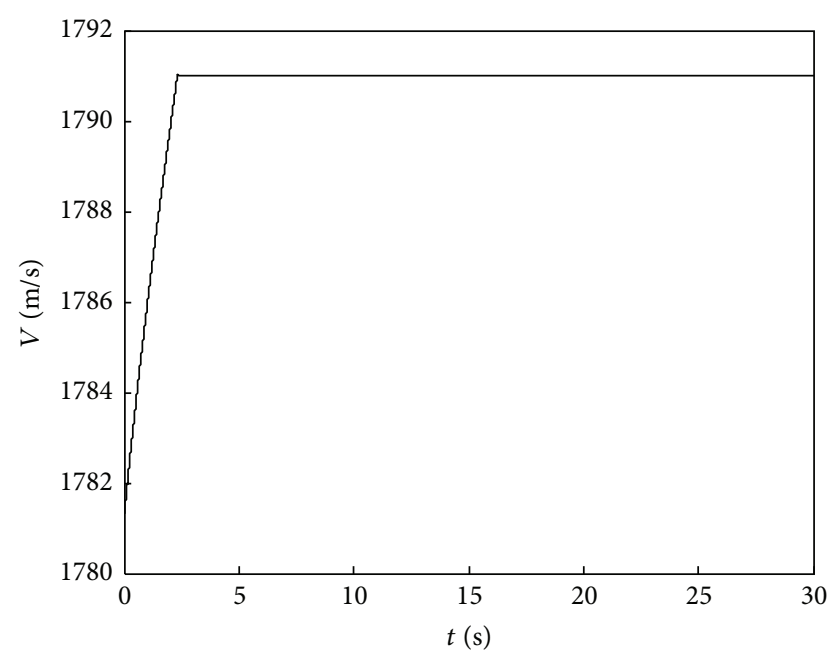

FIGURE 11: Results of velocity.

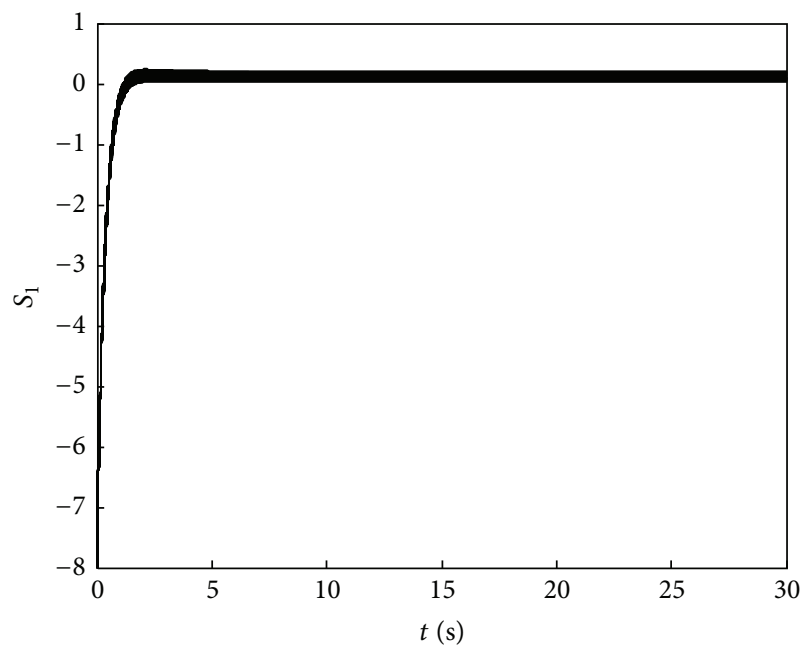

FIGURE 12: Results of sliding surface of acceleration.

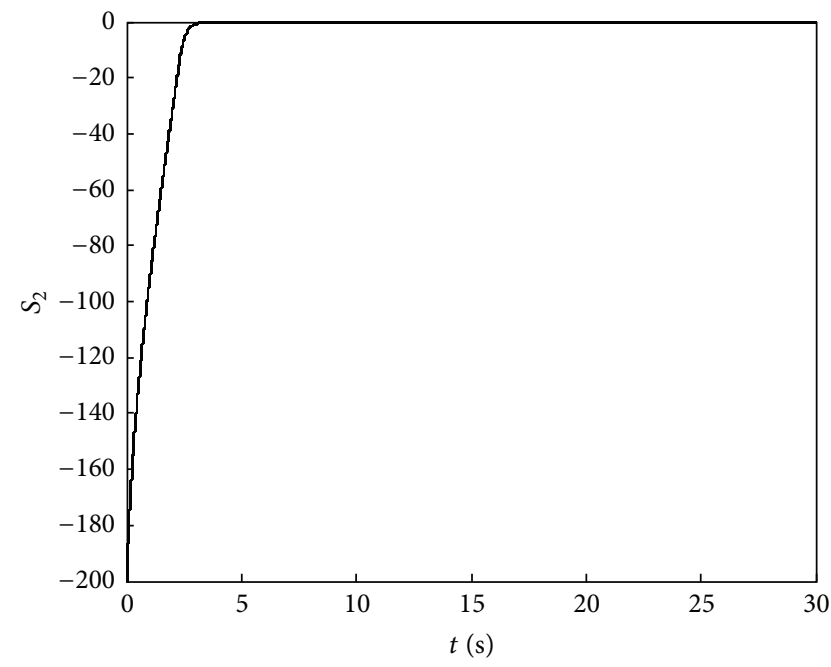

FIGURE 13: Results of sliding surface of velocity. 


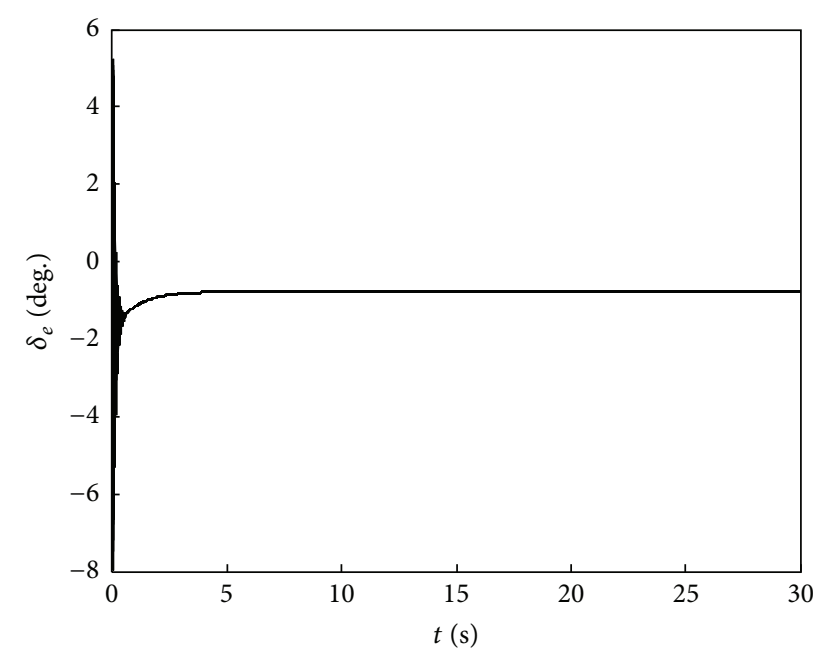

Figure 14: Results of elevator.

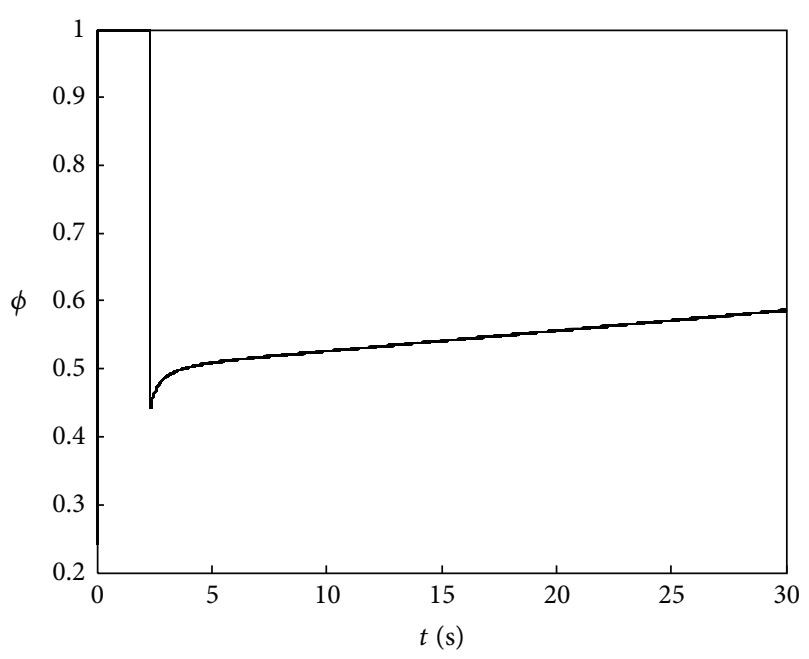

Figure 15: Results of throttle setting.

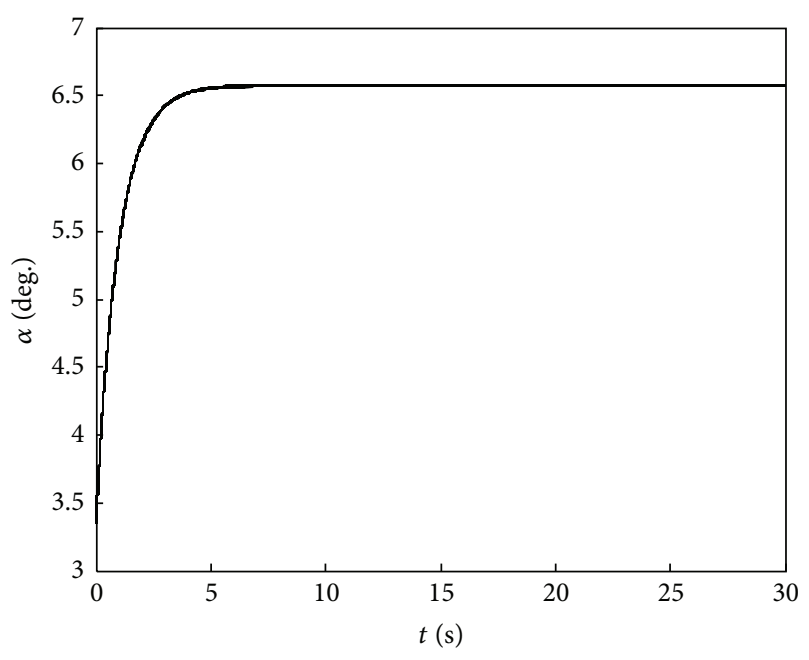

Figure 16: Results of angle of attack.

\section{Acknowledgments}

This paper is supported by Fundamental Research Funds for the Central Universities under Grant 3102015BJ008 and Aeronautical Science Foundation of China under Grant no. 2015ZA53003 and this work is also supported by the Special Science Research Foundation of Doctor Subject for Higher Education under Grant no. 20136102120012.

\section{References}

[1] M. A. Bolender and D. B. Doman, "Nonlinear longitudinal dynamical model of an air-breathing hypersonic vehicle," Journal of Spacecraft and Rockets, vol. 44, no. 2, pp. 374-387, 2007.

[2] Y. H. Fan, Y. F. Yu, and X. Li, "Design of composite control system for an air-breathing hypersonic vehicle with wingrudder deflection," Applied Mechanics and Materials, vol. 719720, pp. 365-368, 2015.

[3] B. Xu, D. W. Wang, H. Wang, and S. Zhu, "Adaptive neural control of a hypersonic vehicle in discrete time," Journal of Intelligent and Robotic Systems, vol. 73, no. 1-4, pp. 219-231, 2014.

[4] H. J. Xu, M. D. Mirmirani, and P. A. Ioannou, "Adaptive sliding mode control design for a hypersonic flight vehicle," Journal of Guidance, Control, and Dynamics, vol. 27, no. 5, pp. 829-838, 2004.

[5] W. Huang, S. B. Li, J. Liu, and Z. G. Wang, "Investigation on high angle of attack characteristics of hypersonic space vehicle," Science China Technological Sciences, vol. 55, no. 5, pp. 14371442,2012

[6] W. Huang, L. Ma, Z.-G. Wang et al., "A parametric study on the aerodynamic characteristics of a hypersonic waverider vehicle," Acta Astronautica, vol. 69, no. 3-4, pp. 135-140, 2011.

[7] Y. Chao, Y. Xu, and C. C. Xie, "Review of studies on aeroelasticity of hypersonic vehicles," Acta Aeronautica et Astronautica Sinica, vol. 31, no. 1, pp. 1-11, 2010.

[8] B. Xu and Z. Shi, "An overview on flight dynamics and control approaches for hypersonic vehicles," Science China Information Sciences, vol. 58, no. 7, 19 pages, 2015.

[9] K. Matthew, "Adaptive control of an aeroelastic airbreathing hypersonic cruise vehicle," in Proceedings of the AIAA Guidance, Navigation and Control Conference and Exhibit, Hilton Head Island, SC, USA, August 2007.

[10] L. Fiorentini, M. A. Bolender, and D. B. Doman, "Nonlinear robust/adaptive controller design for an air-breathing hypersonic vehicle model," in Proceedings of the AIAA Guidance, Navigation and Control Conference and Exhibit, Hilton Head Island, SC, USA, December 2006.

[11] Z. Yang and A. Tian, "A variable structure controller and semi-physical simulation for re-entry hypersonic missile," in Proceedings of the AIAA Guidance, Navigation, and Control Conference, Boston, Mass, USA, August 2013.

[12] P. Yu and S. Yuri, "Adaptive continuous higher order sliding mode control of air breathing hypersonic missile for maximum target penetration," in Proceedings of the AIAA Guidance, Navigation, and Control Conference, Kissimmee, Fla, USA, January 2015.

[13] A. A. Powly and M. S. Bhat, "Missile autopilot design using discrete-time variable structure controller with sliding sector," Journal of Guidance, Control, and Dynamics, vol. 27, no. 4, pp. 634-646, 2004. 
[14] H. H. Choi, "Variable structure control of dynamical systems with mismatched norm-bounded uncertainties: an LMI approach," International Journal of Control, vol. 74, no. 13, pp. 1324-1334, 2001.

[15] B. Xu, "Robust adaptive neural control of flexible hypersonic flight vehicle with dead-zone input nonlinearity," Nonlinear Dynamics, vol. 80, no. 3, pp. 1509-1520, 2015.

[16] B. Xu, C. G. Yang, and Y. P. Pan, "Global neural dynamic surface tracking control of strict-feedback systems with application to hypersonic flight vehicle," IEEE Transactions on Neural Networks and Learning Systems, vol. 26, no. 10, pp. 2563-2575, 2015.

[17] B. Xu, Z. K. Shi, C. G. Yang, and S. X. Wang, "Neural hypersonic flight control via time-scale decomposition with throttle setting constraint," Nonlinear Dynamics, vol. 73, no. 3, pp. 1849-1861, 2013.

[18] B. Xu, D. Wang, F. Sun, and Z. Shi, "Direct neural discrete control of hypersonic flight vehicle," Nonlinear Dynamics, vol. 70, no. 1, pp. 269-278, 2012.

[19] W. B. Gao, Y. F. Wang, and A. Homaifa, "Discrete-time variable structure control systems," IEEE Transactions on Industrial Electronics, vol. 42, no. 2, pp. 117-122, 1995.

[20] J. Wang, H. Van Brussel, and J. Swevers, "Robust perfect tracking control with discrete sliding mode controller," Journal of Dynamic Systems, Measurement and Control, vol. 125, no. 1, pp. 27-32, 2003. 


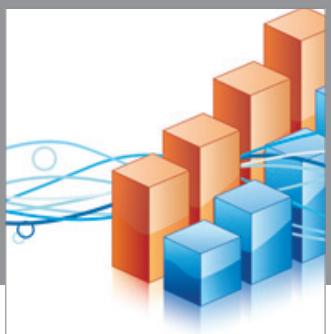

Advances in

Operations Research

vatem alat4

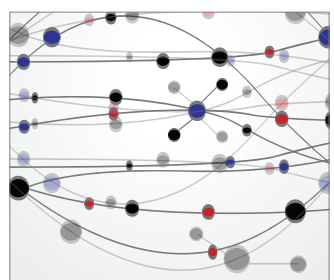

\section{The Scientific} World Journal
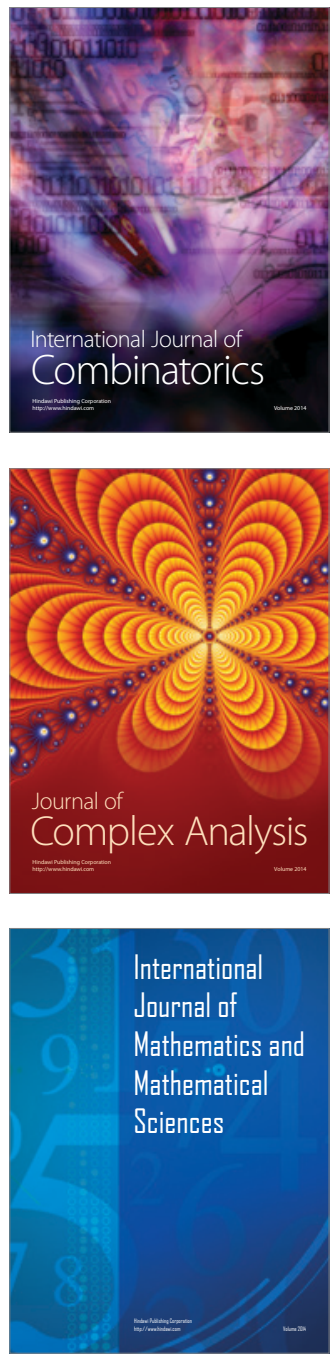
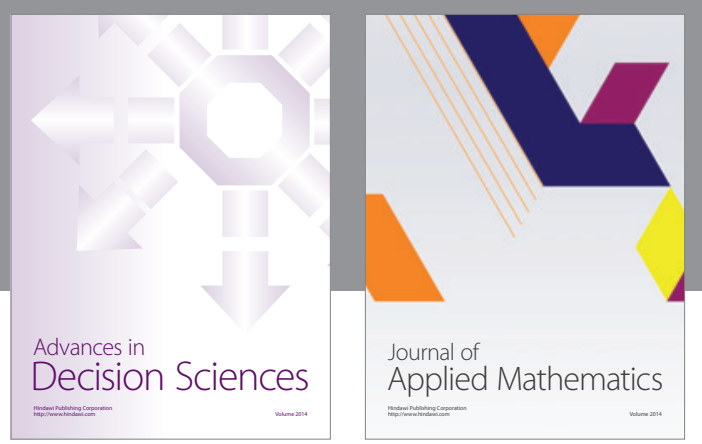

Algebra

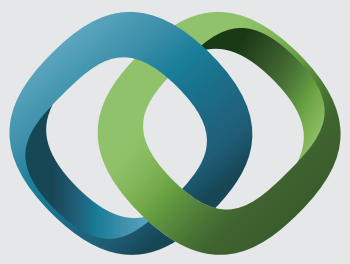

\section{Hindawi}

Submit your manuscripts at

http://www.hindawi.com
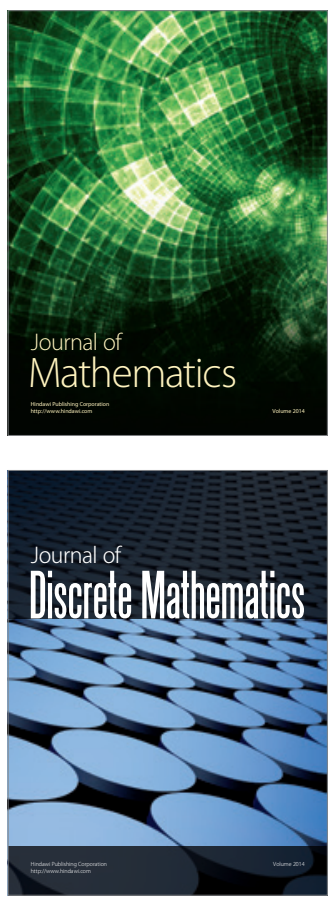

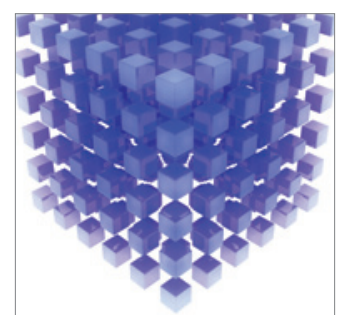

Mathematical Problems in Engineering
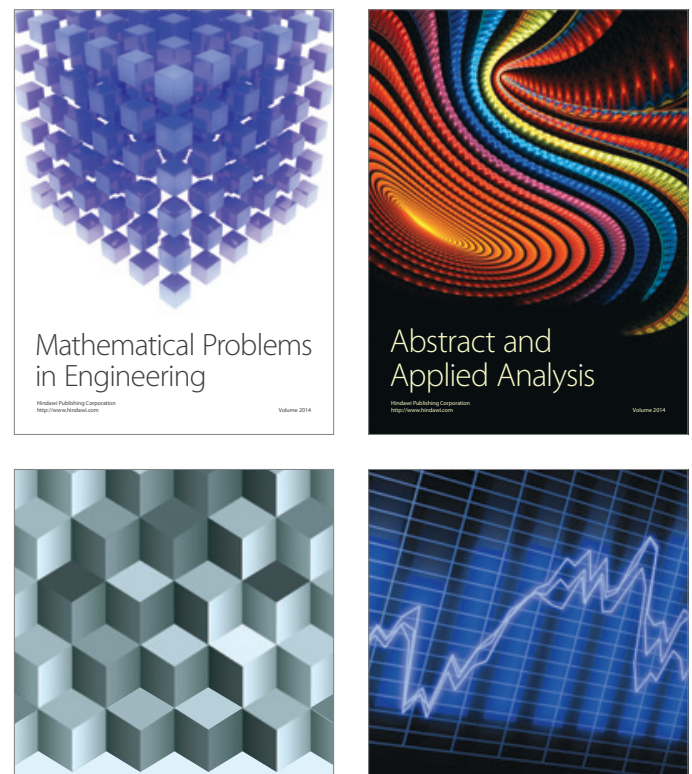

Journal of

Function Spaces

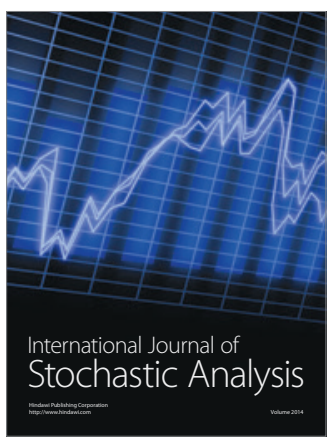

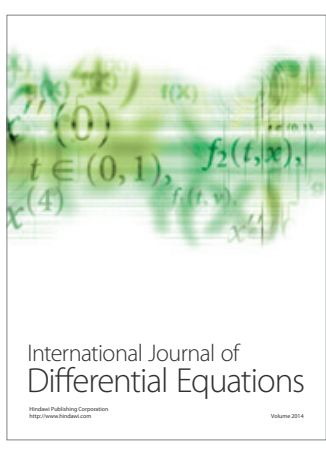
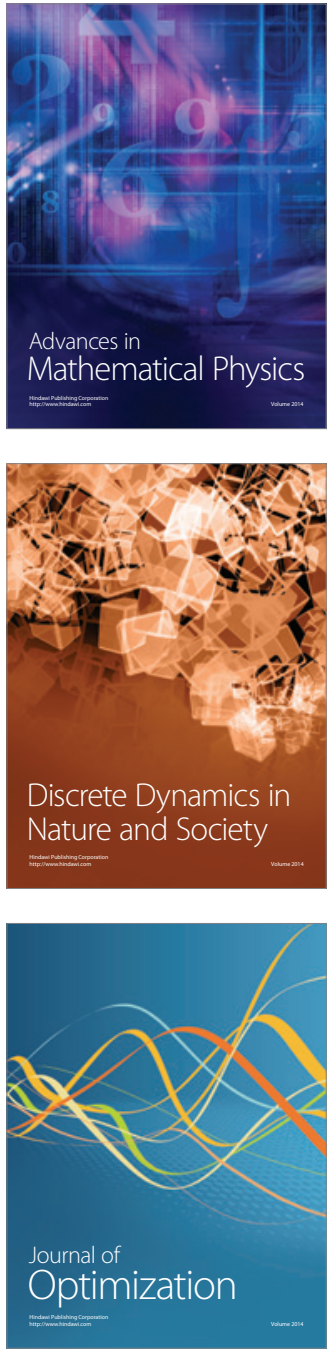